\title{
El espacio de la praxis en Heidegger*
}

\author{
The Space of Praxis in Heidegger
}

\section{O espaço da práxis em Heidegger}

\section{Dr. Francisco Abalo** y Dr. Luis Placencia***}

\begin{abstract}
RESUMEN
En el presente artículo se busca dar cuenta de las peculiaridades metódicas y sistemáticas del enfoque heideggeriano de la espacialidad (Räumlichkeit). De este modo, podrá comprenderse de mejor forma, estimamos, algunas posiciones heideggerianas que han llevado a críticas por parte de sus lectores, especialmente aquella que se relaciona con la pretendida omisión del cuerpo en Ser y Tiempo. Finalmente, nos reapropiaremos de esta crítica clásica, pero desde un sustento diferente al que habitualmente suele servirle de base.
\end{abstract} Palabras clave: espacio, espacialidad, cuerpo propio, Heidegger, Ser y Tiempo

Una primera versión de este texto fue presentada el 30 de agosto de 2018 en marco del III Congreso Nacional de la Sociedad Iberoamericana de Estudios Heideggerianos, sección Chile (SIEH/Chile), realizado en la Pontificia Universidad Católica de Valparaíso. Agradecemos a quienes participaron de la discusión en esa instancia por la ayuda que nos reportaron sus ideas en la mejora del texto. Un lugar especial merecen aquí las valiosas observaciones de Roberto Rubio, tanto en ese contexto como en intercambios posteriores en torno a este texto. En esas instancias posteriores, el Prof. Rubio nos facilitó además importante bibliografía que nos permitió completar la perspectiva del trabajo. El texto ha sido modificado en varios aspectos y en todas sus secciones, entre otras cosas, gracias a las recomendaciones que él nos hizo. Las traducciones son nuestras. El presente artículo se inscribe en el marco del proyecto de investigación FONDECYT/INICIACIÓN n 11170331.

* Chileno, licenciado, magíster y doctor en Filosofía por la Universidad de Chile. Profesor Asistente del Departamento de Filosofía de la Universidad de Chile. Contacto: fjabalo@uchile.cl.

ORCID: https://orcid.org/0000-0002-2470-2811

*** Chileno, licenciado en Filosofía por la Pontificia Universidad Católica de Chile y doctor en Filosofía por la Martin-Luther-Universität Halle-Wittenberg (Alemania). Profesor Asistente del Departamento de Filosofía de la Universidad de Chile. Contacto: luis.placencia@uchile.cl.

ORCID: https://orcid.org/0000-0003-1052-0846 


\begin{abstract}
This article is aimed at revealing the methodical and systematic peculiarities of the Heideggerian approach to spatiality (Räumlichkeit). This will allow us to better understand some Heideggerian positions that have been criticized by his readers, especially that related to the presumed omission of the body in Being and Time. Finally, we reappropriate this classical critique, but from a different supporting foundation than that regularly used as a basis.
\end{abstract}

\section{RESUMO}

No presente artigo buscamos apresentar as peculiaridades metódicas e sistemáticas do enfoque heideggeriano da espacialidade (Räumlichkeit). Deste modo, consideramos que poderão ser compreendidas de melhor forma algumas posições heideggerianas que levaram a críticas por parte de seus leitores, especialmente aquela relacionada com a pretensa omissão do corpo em Ser e Tempo. Finalmente, nos reapropriaremos desta crítica clássica, mas desde uma sustentação diferente da que habitualmente costuma lhe servir de base.
Keywords: space, spatiality, own body, Heidegger, Being and Time

Palavraschave: espaço, espacialidade, corpo próprio, Heidegger, Ser e Tempo 
En el $\S 2$ de la $K r V$ Kant se hace la siguiente pregunta:

“¿Qué son pues el espacio y el tiempo? ¿Son entidades efectivamente reales? ¿Son solo determinaciones o también relaciones de las cosas tales que corresponderían también a ellas incluso si no fueran intuidas? $i \mathrm{O}$ son tales que solo radican en la forma de la intuición y con ella en la constitución subjetiva de nuestro ánimo sin la cual estos predicados no podrían ser agregados a ninguna cosa?" (A 23/B 38).

Como es sabido, Kant toma partido, en su Crítica de la razón pura, por la idea mencionada en el pasaje, junto con otras alternativas que parecieran ser ofrecidas como agotando las posibilidades de interpretar la entidad del espacio y el tiempo, según la cual éstas serían determinaciones de nuestro ánimo, tales que ellos constituirían las condiciones formales de la sensibilidad, i.e. de la capacidad de recibir representaciones al ser afectados por los objetos, sin ser eo ipso propiedades que les corresponderían a los objetos en sí mismos, i.e. con independencia del modo en que ellos son percibidos por nuestra inteligencia finita. Así las cosas, el espacio y el tiempo serían empíricamente reales, pero, a la vez, trascendentalmente ideales, con lo que Kant quiere decir que serían representaciones que poseen validez para todos los objetos de la experiencia, pero solo en cuanto objetos de la experiencia (A 28/B 44). De este modo, se podría expresar el corazón de la posición kantiana empleando una formulación que no por paradójica deja de ser correcta, sc. Kant funda el carácter "objetivo" del espacio en su carácter "subjetivo" ${ }^{2}$. Por último, cabe destacar en este contexto que la doctrina anterior es, como se puede apreciar de modo notorio en el $\S 3$ de la $K r V$, fundamento principal de la distinción crítica entre fenómenos y cosas en sí (A 27/B 43). Dicho de otro modo: al mismo tiempo que la concepción kantiana del espacio funda su carácter objetivo (realidad empírica) en su rasgo de ser una condición subjetiva de nuestra capacidad representativa, queda también establecida su estatus de mera condición subjetiva (idealidad trascendental) y por ello su carácter de condición de los meros fenómenos, no así de las cosas 
tal como ellas serían percibidas con independencia de nuestras condiciones subjetivas.

Como también es sabido, las consideraciones kantianas sobre el origen subjetivo de la validez de la esfera espacial inspiraron fuertemente a autores como Husserl en su concepción del espacio, así como también en su intento de dar cuenta del origen de formas de saber vinculadas a él, como la geometría. De este modo, no es de extrañar que la posición en torno al espacio que sostiene Heidegger, un filósofo que, como sabemos, construye sobre la base de una fuerte injerencia tanto de Husserl como de Kant, pueda ser leída con sentido como un intento de dar cuenta de modo distinto de la tensión aparentemente paradojal entre los elementos "subjetivos" y "objetivos" que implicaría el carácter espacial de la experiencia, mas todo esto a partir de un marco metódico y sistemático radicalmente nuevo que, en la medida en que se apropia críticamente del enfoque trascendental que representan los dos autores clásicos de esta tradición (Kant y Husserl), le da a estos elementos "subjetivos" y "objetivos" una significación que ya no puede ser más mentada por esos términos, so pena de un desdibujamiento total del significado habitual de los mismos. Este enfoque superador tiene como consecuencia una nueva y original concepción del carácter $a$ priori del espacio, que pareciera no entrañar las dificultades que un planteo como el kantiano trae consigo, v.gr. una peligrosa cercanía con el psicologismo o el establecimiento de la problemática distinción entre "fenómeno" y "cosa en sí".

En lo que sigue intentaremos dar cuenta, al menos parcialmente, del modo en que Heidegger sostiene este planteo, que pretende superar la concepción de cuño kantiano en lo tocante al carácter a priori del espacio, así como de las peculiaridades metódicas y sistemáticas del enfoque heideggeriano de la espacialidad (II). Esto nos permitirá comprender de mejor modo las razones que están detrás de la que para muchos autores es una de las debilidades fundamentales del planteo heideggeriano en Ser y Tiempo (en adelante SZ), sc. la omisión de un tratamiento de la dimensión corporal del Dasein. Finalmente, luego de comprender las razones que llevan a Heidegger a no considerar la cuestión de la corporalidad - al menos en los textos en los que trata del problema del espacio-, nos reapropiaremos de esta crítica clásica, pero desde un sustento diferente al que habitualmente suele servirle de base (III). 


\section{II}

Los lugares más visitados en los que M. Heidegger expone su concepción sobre la espacialidad se hayan principalmente en SZ. Se trata de los célebres parágrafos 22 a 24, contenidos en el tercer capítulo de la primera sección (El análisis fundamental preparatorio del Dasein), y el parágrafo 70 del cuarto capítulo de la segunda sección (Dasein y temporalidad), ambas secciones contenidas en la única parte publicada del tratado. No son, con todo, los únicos en los que Heidegger se refiere a esta cuestión y a temáticas afines. Sin embargo, un estudio que analice la cuestión en cada uno de los distintos textos en que Heidegger acomete el asunto mencionado y muestre las relaciones sistemáticas y genéticas entre ellos, no puede ser abordado en el largo de un artículo. De acuerdo con nuestro conocimiento, un trabajo de este tipo es, de hecho, una tarea pendiente todavía para la scholarship heideggeriana. Por esta razón, este texto se dedicará a una tarea más modesta, pero que puede servir de primera piedra para un trabajo como el anteriormente mencionado. Se tratará fundamentalmente de abordar el tratamiento heideggeriano de la espacialidad en el contexto del opus magnum de Heidegger con alguna alusión acotada a obras muy cercanas en el tiempo ${ }^{2}$. Nuestro interés, en consecuencia, estará centrado en un análisis de algunos detalles de la sección que no han sido, a nuestro juicio, rectamente remarcados por la literatura secundaria. Por la misma razón tampoco nos detendremos en los todos los elementos relevantes del argumento de la sección.

Un primer punto que debe hacerse notar es que, en el marco de la investigación especializada, la concepción contenida en estos parágrafos ha recibido una atención, aunque creciente, relativamente menor comparada con otros tópicos que, al menos prima facie, tienen una relevancia sistemática más notoria. El caso más patente es el que concierne al tiempo. Pareciera haber buenas razones para ello. En efecto, como ya se mencionó, son pocos los parágrafos que Heidegger dedica a la cuestión de la espacialidad, estando además uno de ellos consa-

\footnotetext{
2 En efecto, algunas indicaciones valiosas en relación al tópico que aquí nos interesa se pueden hallar en Prolegómenos para una historia del concepto de tiempo (GA 20 \$25, 306 y ss.), que es, como se sabe, un texto sumamente próximo, tanto en el contenido como en el tiempo, a SZ (Kisiel 197). Aquí las tendremos en cuenta solo ocasionalmente, pues el foco estará pues en SZ.
} 
grado a mostrar la dependencia de la espacialidad de la temporalidad extático-horizontal. Con todo, basta con poner una mínima atención a la morfología de la palabra mediante la cual se designa al ente "que somos en cada caso nosotros mismos" (Dasein), y que está en el centro de la investigación de SZ, para sospechar al menos que la concepción del espacio en SZ tiene un mayor alcance y relevancia de lo que un número acotado de parágrafos, comparativamente hablando, parece indicar a primera vista ${ }^{3}$.

La mayoría de las interpretaciones de esta concepción, sean reconstructivas o críticas, han estado dominadas por tres aspectos relevantes. En primer lugar, es habitual en las reconstrucciones de estos parágrafos, no sin justa razón, la tendencia a realzar los aspectos en que dicha concepción es máximamente distante de una concepción geométrica ${ }^{4}$, tal como está documentada en esa forma extrema de ontología de objetos que Heidegger atribuye a Descartes. En este sentido, son célebres los parágrafos inmediatamente anteriores a la presentación de la cuestión de la espacialidad $(\$ \S 19-21)^{5}$. Por otro lado, gran parte de las críticas (v.gr. Aho y Franck) consideran relevante para la evaluación de esta concepción la autocrítica que Heidegger esbozara años más tarde a la tesis que aparece en §70, según la cual “Sólo sobre la base de la temporalidad extático-horizontal es posible la irrupción del Dasein en el espacio" (369) $)^{6}$. Vinculada a esta posibilidad de distanciamiento crítico, aparece a su vez la observación asimismo crítica a la proverbial omisión en SZ de un tratamiento más amplio del cuerpo que la escueta mención de éste en el §23 (108)

\footnotetext{
3 Existen otros pasajes que destacan la conexión entre la espacialidad y conceptos centrales de SZ como "ser-en-el-mundo" o aperturidad. Cf. §12, 56; §60, 299. Heidegger mismo destaca en SZ la remisión que habría en el término Dasein al Da que "según el sentido familiar de la palabra apunta a "aquí» y «allá»"

4 Dreyfus (139) presenta una útil contraposición de las diferentes características de las formas de espacio que contrapone Heidegger, concretamente en el §24. Siguiendo en alguna medida lo establecido por Dreyfus, podríamos caracterizar lo que aquíllamamos "geométrico" como siendo homogéneo, sin centro, pura extensión, tridimensional, compuesto de un agregado de una multiplicidad de posiciones posibles y medible.

5 Este aspecto lo hacen notar con fuerza autores como Villela-Petit (122), Vallega (94111) y Cerbone (130).

6 En concreto, sostiene Heidegger en su conferencia "Tiempo y ser" de 1962, "el intento en el $\$ 70$ de Ser y Tiempo de reconducir la espacialidad del Dasein a la temporalidad no se sostiene" (GA 14, 29).

7 "La espacialización del Dasein en su «corporeidad», que trae consigo una problemática propia que no ha de ser tratada aquí, está distinguida según estas direcciones".
} 
En esta parte de la presente exposición no nos orientaremos primordialmente por estas consideraciones predominantes en la literatura especializada, sino que intentaremos hacer énfasis en al menos dos aspectos que no han sido suficientemente remarcados y que constituyen, a nuestro juicio, elementos claves para la comprensión heideggeriana del espacio a la altura de SZ. Esta estrategia, por otra parte, no aspira a inmunizar de toda crítica a la concepción contenida en los parágrafos mencionados, sino establecer el terreno para una crítica de otro orden, que será presentada en la sección que sigue. Con todo, permítasenos, antes de realizar ese ejercicio, que dejaremos para la sección final, ponderar previamente de mejor modo la observación crítica que los objetores de Heidegger suelen alegar con mayor agudeza, sc. la de la omisión de un tratamiento de la corporalidad del Dasein. Nos parece que, rectamente comprendidas las motivaciones de Heidegger, esta omisión es en buena medida explicable. Partiremos, sin embargo, con un problema que en principio puede parecer meramente formal, pero que nos abrirá el camino para la consideración que aquí queremos hacer.

En varios lugares Heidegger habla de una "espacialidad existencial" (existenziale Räumlichkeit/ §12, 54). A primera vista, parece razonable identificar lo significado a través de esta frase con lo que Heidegger denomina también "espacialidad del Dasein" (Räumlichkeit des Daseins/ $\$ 24,110 ; \S 70,367)$, y lo que también nombra como "espacialidad del seren-el-mundo" (Räumlichkeit des In-der-Welt-sein/\$23, 104). Con todo, el asunto es más complicado. Como es sabido, la espacialidad de este ente se distingue netamente del ser en el espacio dicho del ente en la modalidad de mero estar presente (Vorhandenheit), que Heidegger denomina "interioridad" (Inwendigkeit), por una parte, y del ente en la modalidad del estar a la mano (Zuhandenheit), cuya forma específica de espacialidad es la de la pertenencia a su lugar (plazierbare Hingehörigkeit), por otra $^{8}$. Como se sabe también, la espacialidad existencial es primaria en relación con las otras dos desde un punto de vista de relaciones de fundación ${ }^{9}$. Aun cuando ellas no sean del todo claras en ambos casos, al

\footnotetext{
$8 \quad$ "La espacialidad al Dasein, que esencialmente no es un estar-ahí, no puede significar algo como aparecer en una posición «en el espacio del mundo» ni estar a la mano en un lugar" $(\$ 23,104)$.

$9 \quad$ "El dejar comparecer de lo a la mano en su espacio circundante sólo es ónticamente posible porque el Dasein mismo es «espacial» con miras a su ser-en-el-mundo" $(\$ 22,104)$.
} 
menos sí se puede decir que buena parte del $\$ 24$ está dedicado a esbozar en qué medida el conocimiento del espacio y, por tanto, la comprensión "geométrica" del mismo depende en último término de la espacialidad existencial en el sentido del "dar lugar" (Einräumen $)^{10}$. Ahora bien, si este es el caso, ¿ ¿ómo puede el autor expresar el siguiente pensamiento: "El aquí [del Dasein] lo comprende el Dasein desde el allí del mundo circundante" (Sein Hier versteht das Dasein aus dem umweltlichen Dort $\$ 23,107)^{11}$, lo cual parece implicar, prima facie, el orden inverso de dependencia? La respuesta satisfactoria a esta pregunta supone, a nuestro entender, considerar dos aspectos sistemático-metódicos que condicionan de punta a cabo la concepción aquí expuesta.

En primer lugar, aquello a lo que refiere la expresión "espacialidad existencial" no apunta directamente a la pregunta sobre qué sea aquello que permite que un individuo pueda determinar el lugar en el que de hecho se halla. Si la expresión "espacialidad del Dasein" admite un sentido en el que se trata del "aquí" que en cada caso un individuo ocupa en el mundo y que puede ser determinado según ciertos criterios, la "espacialidad existencial" apunta a algo más amplio que implica lo anterior, pero que no se agota en ello. Visto desde un punto de vista metódico, la espacialidad existencial es el carácter espacial de algo visto desde el punto de vista de una cierta estructura ontológica: la existencia. Esto es clave, pues precisamente lo que dentro del análisis de la espacialidad llamará Heidegger "existenciales" (la desalejación, la direccionalidad) no son sino elementos explicativos de aquello que tenemos a la vista cuando decimos, por ejemplo, que algo "está en un lugar", elementos que a su vez provienen de una cierta idea de existencia que el autor ha anticipado ya en el $\$ 9$ (41-45). Para nadie que haya leído el texto es un misterio que la idea de existencia en SZ es sui generis, pues implica eminentemente un carácter proyectual. Ya en la descripción formal de existencia, según la cual el ente del caso existe en la medida en que le "va su ser", que existir es "tener que ser", desliza este aspecto $(\$ 9,42)$. Así, por ejemplo, eso a lo que nos referimos de forma intuitiva, i.e. sin fijar temáticamente el dominio de este concepto a través de una definición o a un recurso de

10 Cerbone, con buenas razones, ha sido especialmente crítico de la tesis heideggeriana relativa posibilidad de derivación del "espacio de la naturaleza" a partir de la "espacialidad existencial”. Cf. Cerbone 2013, 142-143.

$11 \quad$ Cf. $\$ 28,132 ; \S 70,368$ 
este estilo, cuando hablamos de "acciones", documenta algún tipo de posibilidad de realización de sí mismo que, habiendo sido asumida de forma más o menos consciente, más o menos responsable, ha "lanzado" al agente más allá de su acción considerada como un hecho actual. Es decisivo este aspecto en el presente contexto pues es, en parte, bajo el aspecto proyectual que Heidegger considerará el peculiar carácter móvil (Bewegtheit, §72, 375) de ese ente que tiene la forma de ser de la existencia. ¿Por qué decimos esto?

Ciertamente, buena parte de los comentadores ha hecho hincapié en que la situación fenoménica desde donde se intenta reconstruir la concepción de la espacialidad se documenta en la experiencia pragmático-operativa propia de un agente y no la constativo-perceptiva de un observador. Pero a lo que no se le ha sacado suficientes rendimientos es al hecho de que, en tanto se trata de la agencia, el problema aquí tratado no se refiere solo a las condiciones de posibilidad de la aparición de objetos (útiles) en el espacio (Dreyfus), sino efectivamente a la ocupación dinámica que el agente hace del "espacio". En otras palabras, al apelar de forma más bien intuitiva a la acción humana, Heidegger espera documentar aquellos elementos que explican, desde el punto de vista de la idea de existencia antes señalada, la peculiar forma que tiene un ente de "abrir" dinámicamente espacio. "Desalejación" y "direccionalidad" son los nombres para esos explicantia de la apertura dinámica que un ente hace del espacio, solo si se asume, por cierto, que ese ente tiene la forma de ser de la existencia ${ }^{12}$.

Ahora bien, esto solo aborda parcialmente el problema antes señalado. El otro elemento metódico-sistemático que hay que considerar es el segundo aspecto formal contenido en la idea de existencia. Para un

12 "El Dasein, sin embargo, es «en» el mundo en el sentido del ocupado y familiar habérselas con el ente que se encuentra en el mundo. En consecuencia, si le corresponde en alguna medida espacialidad, entonces es esto sólo posible sobre la base de este seren, cuya espacialidad muestra el carácter de la desalejación y la direccionalidad" ( $\$ 23$, 104-105). En relación con el carácter de apertura dinámica del espacio ha de destacarse que no es casual que la desalejación al menos, que por otra parte es el existencial relativo al espacio en cuya explicación Heidegger invierte más espacio, sea caracterizada en términos transitivos: "usamos la expresión desalejación en un sentido activo y transitivo" ( $\$$ 23, 105). La "direccionalidad" parece ser caracterizada por Heidegger como "correspondiendo" o "perteneciendo" a la "desalejación" y, por tanto, ya entrañando este elemento activo: "Queda por atender el que la direccionalidad que pertenece a la desalejación está fundada en el ser-en-el-mundo" (\$23 109). 
ente, "existir" significa estar o ser en el mundo, en donde "mundo" no es un conjunto infinito de objetos considerados extensionalmente, sino un momento estructural (la articulación de significatividad) que hace parte de la existencia de un ente. Es decir, el carácter proyectual de la existencia implica un horizonte de articulación de funciones teleológicas (para-qué, el para-eso, el por mor de), las cuales algunas son satisfechas por entes que no tienen la forma de ser de la existencia, pero cuya estructura ontológica implica posibilidades de ese ente que tiene la forma de la existencia. Es la idea que se documenta en la consideración de que los objetos aparecen como plexos de útiles (y no primeramente útiles individuales) que exhiben "de suyo" un potencial de utilización para una acción que virtualmente un agente requiera emprender con ellos. Nuevamente, al apelar de forma más bien intuitiva a nuestra acción (al trato pragmático-operativo con el ente), Heidegger intenta mostrar que para que a un ente (que tenga la forma de ser de la existencia) le puedan salir al encuentro objetos (en la modalidad inmediata de cosas con las que una posible acción requiere realizarse), tiene que suponerse como haciendo parte de su propia estructura ontológica un horizonte de significatividad ya abierto, aun cuando de facto no esté inmediatamente presente para él.

Esta concepción del mundo (horizonte significativo), como momento estructural de la existencia, es decisiva para calibrar en qué medida el auténtico punto de partida metódico de los análisis acerca del espacio está concentrado en la afirmación, que sin embargo solo aparece hacia el final del tratamiento heideggeriano, según la cual "sólo retornando al mundo es posible comprender el espacio" ( $(24,113)^{13}$. Es cierto que, en el decurso argumentativo de estos parágrafos, el contraste con la concepción "geométrica" del espacio tiene una gran relevancia, como se ha enfatizado de manera insistente por la literatura y, por cierto, con razón, tal como ya se indicó. Pero la relevancia de la polémica contra la versión "objetiva" del espacio hace que se atenúe o se pierda de vista el intento de distanciamiento frente a otra posibilidad: la reducción de la espacialidad a elementos puramente subjetivos, posibilidad que el autor identifica con el nombre de Kant ${ }^{14}$.

\footnotetext{
$13 \quad C f$., además, $\S 12,56 ; \S 21$ 101-102.

14 “El espacio no está ni el sujeto ni está el mundo en el espacio" $(\S 24,111)$. "Derecha e izquierda no son algo «subjetivo» para lo que el sujeto tenga un sentimiento, sino que
} 
Este complejo equilibrio con el que Heidegger pretende combatir tanto la tentación "objetivista" como la "subjetivista" en lo que se refiere al tratamiento del espacio es presentado de modo compacto en el siguiente pasaje que, además, destaca las consecuencias filosóficas que tiene lo anterior:

El espacio no está ni el sujeto ni está el mundo en el espacio. El espacio está más bien «en» el mundo en cuanto el estar-en-el-mundo constitutivo para el Dasein ha abierto espacio. El espacio no se encuentra en el sujeto ni considera éste al mundo «como si» estuviese en un espacio, sino que el «sujeto» ontológicamente bien entendido, el Dasein, es en un sentido originario, espacial. Y porque el $D a-$ sein es espacial en el sentido descrito se muestra el espacio como $a$ priori. Este título no dice algo así como la pertenencia precedente a un sujeto que aún no tiene mundo, que arroja un espacio desde sí. A prioridad dice aquí: precedencia del encuentro del espacio (como zona) en el encuentro cada vez circunspectivo de lo a la mano (\$24 111).

Dicho de otra forma, si es verdad que Heidegger tiene por punto de contraste tanto la comprensión "objetiva" del espacio como la "subjetiva", entonces los explicantia existenciales de la espacialidad no pueden ser considerados como operaciones o disposiciones de un sujeto, sino como elementos explicativos que pertenecen a una estructura (la existencia) que implica aspectos, digámoslo así, no reductibles a la subjeti-

son las direcciones de la orientación a un mundo cada vez ya a la mano” ( $\$ 23109)$. Este último pasaje remite de modo explícito a la idea que Kant desarrolla en el texto - citado por Heidegger- “¿Qué significa orientarse en el pensamiento?", así como en el más temprano y no referido por nuestro autor "Del fundamento primero de la distinción de las regiones en el espacio" (Von dem ersten Grunde des Unterschiedes der Gegenden im Raum, cfr. AA 02 375-383). Es de destacar que estos textos presentan claras afinidades con el modo en como trata el problema del espacio Husserl en Ding und Raum, o del cuerpo propio como "punto cero" de las orientaciones en Ideas II (Hua IV, 158). Cf. Placencia (60). No es irrelevante enfatizar en este punto que, ya en Prolegómenos, Heidegger parece oponerse tanto a la consideración "objetivista" (Descartes) como "subjetivista" (Kant) del espacio, quedando ambas, a partir de esta concepción, en la misma vereda. La razón, si mal no entendemos, estriba en que en ambos casos se reconstruye la noción de espacio unilateralmente a partir de uno de los dos lados de la relación sujetoobjeto. Si alguna pretensión tienen los análisis sobre la "espacialidad", una vez que se toma en cuenta que el correcto punto de partida es el ser-en el- mundo, es justamente el de superar la unilateralidad que supone todo reconstrucción que se base en el esquema sujeto-objeto. Cf. Prolegómenos para una historia del concepto de tiempo (GA 20) §25, 307, 320ss. 
vidad $^{15}$. Esta irreductibilidad entraña, por un lado, el que la estructura ontológica de la "existencia" trae consigo la remisión a un horizonte que, en el caso de la espacialidad, se documenta de manera clara en lo que denomina Heidegger la "zona" (Gegend) o, dicho con mayor precisión, el carácter zonal del emplazamiento de contextos de útiles que le salen al encuentro a un ente que abre dinámicamente (desalejando y direccionadamente) el espacio. Este carácter zonal de los lugares propios en que se ubican los útiles es irreductible a un objeto en el espacio, pues está ya predescubierto (vorendeckt) en todo dirigirse a contextos de útiles para ponerlos a disposición, según una acción que se pretende llevar a cabo con ellos. La zona es, en este sentido, un momento de los elementos a priori que constituye, en conjunto con los existenciales señalados más arriba, las condiciones de posibilidad de aparición de un objeto espacial. De esta forma, los elementos explicativos fundamentales de la espacialidad, vista desde el punto de la existencia, son la desalejación, la direccionalidad y el carácter zonal de los lugares propios (GA 20, §25, 308).

Ahora bien, los aspectos aquí presentados en general permiten comprender por qué este tratamiento no implica una inconsistencia. La espacialidad existencial, si entendemos por esto la concepción del espacio que toma como punto de vista esa estructura ontológica llamada "existencia", constituye un nivel de análisis más básico que el que supone la respuesta a la pregunta por aquello en función de lo cual es posible determinar el lugar que de facto ocupa el Dasein, aun cuando lo primero contenga aquello que permite responder a lo segundo. De hecho, es justo la espacialidad de la existencia la que permite entender que la espacialidad de Dasein, es decir, la posibilidad de fijación del lugar que de facto ocupo, no supone necesariamente, a juicio de Heidegger $(\$ 23,108)$, la conciencia expresa de mi propio cuerpo, sino que los puntos de referencia que permiten determinar mi "aquí" son externos, es decir, se constituyen a partir de plexos uti-

15 Este punto cobra particular relevancia, especialmente a la luz de las críticas de Dreyfus a la concepción que Heidegger presenta en los parágrafos 22-24. En efecto, Dreyfus estima que, en determinados momentos, Heidegger mismo pierde de vista la distinción "de la apertura general del espacio como campo de presencia (Desalejación) que es la condición para que las cosas estén cerca o lejos de con el traer las cosas cerca del Dasein y el usarlas" (Dreyfus 132). Para una respuesta a las críticas de Dreyfus, se puede ver los trabajos de Arisaka y Cerbone. 
litarios emplazados en zonas predescubiertas. El "aquí" que de facto ocupo es en cada caso relativo, relativo a aquello que requiero para poder llevar una acción asumida como propia. Pero precisamente la fijación del aquí en estos términos supone, por una parte, una ocupación desalejante y orientada del espacio, y, por otra, un mundo que ya se ha abierto ahí en la forma de zonas para esta orientación pragmática. Con ello parece quedar fundada la "desatención" de parte de Heidegger a la cuestión del cuerpo, al menos en los parágrafos en comento.

\section{III}

Con todo, el asunto es más complicado. Incluso a la luz de la recta consideración del tratamiento heideggeriano que se acaba de hacer, cabe replantear la cuestión de la omisión de la referencia al cuerpo. Para entender por qué es necesario, nuevamente un pequeño rodeo.

Anscombe escribe en el $\S 4$ de Intention:

Ahora, parece obvio que en general la pregunta de cuáles son las intenciones de alguien es sólo decidida con autoridad por él. Una razón para esto parece ser que, en general, no sólo estamos interesados en la intención de alguien de hacer lo que él hace, sino en su intención al hacerlo. Usualmente esta última no puede ser vista a partir del mero ver lo que él hace. Otra razón es que en general la pregunta por si alguien intenta hacer lo que él hace simplemente no surge (porque la respuesta es obvia). Y si surge, ella se responde, usualmente, preguntándole. Además, alguien puede formar una intención en la que no persevera, sea porque es impedido por algo o porque sencillamente cambia de opinión. La intención misma, sin embargo, puede ser completa, aunque quede como una cosa puramente interior. Todo esto lleva nos lleva a la idea de que si queremos conocer las intenciones de alguien debemos investigar los contenidos de su mente, y solamente ellos. Y, en consecuencia, nos lleva a pensar que si queremos entender lo que es la intención debemos investigar algo cuya existencia se da en la pura esfera de la mente y a pensar que, aunque la intención se manifiesta en acciones y la manera en que esto ocurre también presenta preguntas interesantes, lo que ocurre físicamente, i.e. los que uno realmente hace, es lo último que necesitamos considerar en nuestra investiga- 
ción. Por el contrario, yo quiero decir que es el primero (Anscombe 2000 9).

Si lo dicho sobre Heidegger es correcto, entonces la observación de Anscombe parece ofrecer una perspectiva de acuerdo con la cual la omisión de la problemática del cuerpo en la filosofía de Heidegger y, en concreto, en la sección en la que éste trata su concepción del espacio, no se muestra ya como una cuestión baladí ni meramente externa al punto de vista metódico y sistemático adoptado por él mismo. Tratemos de elucidar esta cuestión: según habíamos dicho, el hecho de que Heidegger intente tematizar en su análisis de la espacialidad una concepción que no es la que se privilegia en una experiencia constatitvo-preceptiva, sino la que supone una de corte pragmático-operativa, entraña una apelación, implícita por cierto, al orden la acción humana. En efecto, como es sabido el estudio fenomenológico del momento "mundo" que se realiza como parte del análisis del "estar-en-el-mundo" se lleva a efecto a partir del estudio de la experiencia "inmediata y regular", particularmente con el ente que tiene el modo del ser útil, modo de ser a partir del cual será derivado el de aquello que está "ahí delante". No parece haber posibilidad de pensar esta relación al útil sin apelación, aunque sea implícita y sin una caracterización explícita de ese orden, al ámbito de la acción humana. Es la remisión a esta misma experiencia "inmediata y regular" con el ente que tiene el modo de ser del útil la que permitió a Heidegger remontarse a la "espacialidad" de la existencia como genuino elemento a priori y, por ello, como "condición de posibilidad" de la experiencia que hace cada Dasein de su propio cuerpo, i.e. de su lugar en el mundo. A partir de este orden de las acciones, adquieren sentido la "desalejación" y la "direccionalidad" como lo que ha de ser tematizado en un tratamiento del espacio a partir del modo en que éste es experimentado o, si se quiere, abierto por un ente que tiene el modo de ser del Dasein. Dicho de otra forma, el orden de la acción es una especie de presupuesto en el tratamiento heideggeriano del espacio. Con todo, si seguimos la pista que nos da Anscombe, no parece sencillo hacer sentido de este orden sin tener en cuenta el hecho de que la acción misma presupone el cuerpo. Veamos este punto con calma.

El primer paso para comprender este punto viene dado por la comprensión que hemos ganado del término "acción" a partir de la inves- 
tigación de autores como la misma Anscombe o Davidson. Ambos autores, más allá de sus enormes diferencias, compartían la idea de que algo ha de ser llamado "acción" en la medida en que es "intencional bajo una descripción" (Anscombe 2000 11-12 Anscombe 1979 210 Von Wright 91 Davidson 46). Esta idea entraña un descubrimiento poderoso que tiene, al menos, una doble faz. En primer lugar, implica que ser acción intencional es algo que corresponde a algo dependiendo del modo en que ello sea descrito y no como una "propiedad" que le corresponda, por así decir, desde cualquier perspectiva. En otras palabras, "ser intencional" no es, puesto en el lenguaje de la filosofía analítica contemporánea, una "propiedad" de un evento o de algún ítem realmente existente (Davidson 46). En segundo lugar, el descubrimiento trae consigo la idea de que la noción misma de la acción es dependiente de la de intención ${ }^{16}$. Nada es acción si no es "intencional bajo una descripción". Nada de lo dicho por Heidegger parece ser inconsistente con esta idea, sino que más bien, al revés, la mera idea del carácter derivado del ente "ahí delante" a partir del ente "a la mano" parece estar en acuerdo con ella. Ahora bien, la pregunta clave entonces es si el orden de la acción, i.e. ese orden que solo es comprensible a partir de aquel de la intención, es aprehensible sin remisión a un orden de relaciones entre objetos, i.e. al orden de "lo que ocurre físicamente", en el decir de Anscombe. Ésta parece querer sostener que no, i.e. parece querer decir que el orden al que pertenece la intención no es tanto el orden de aquello que se manifiesta en la pura "interioridad" del agente, sino el de aquello que efectivamente ocurre. Solo a partir de él sería legible la intención de un agente ¿Cuál es la razón para ello?

En el $₫ 23$ de Intention, Anscombe nos propone considerar la siguiente situación:

Alguien está bombeando agua a una cisterna que provee de agua potable a una casa. Alguien ha encontrado la forma de contaminar sistemáticamente la fuente con un veneno acumulativamente fatal cuyos efectos no pueden ser notados por quienes beben el agua hasta que ya no pueden curarse. La casa es habitada usualmente por un pequeño grupo de jefes de un partido con sus fa-

16 Esta idea ha sido contradicha por algunos autores, aunque, a nuestro juicio, no de modo sólido. Cfr. Hyman. 
miliares cercanos, que controlan un gran estado. Ellos quieren exterminar a los judíos o quizás planean una guerra mundial. El hombre que contamina la fuente ha estimado que si estas personas son destruidas buenos hombres llegarán al poder, gobernarán bien y quizás instaurarán el reino de Dios en la tierra y asegurarán una buena vida para todos. Él ha revelado su estimación junto con los datos sobre el veneno al hombre que bombea (...) los brazos del hombre se mueven de arriba a abajo. Ciertos músculos, con nombres latinos conocidos a los doctores, se contraen y se relejan. Ciertas sustancias se generan en algunas fibras nerviosas - sustancias cuya generación en el movimiento voluntario interesan a los fisiólogos (...) el bombeo hace una serie de ruidos que suenan como un ritmo conocido (...) Ahora preguntamos: ¿qué está haciendo este hombre? ¿cuál es la descripción de su acción? (Ancombe 2000 37).

Una forma elemental de responder a esta pregunta sería, como sugiere Anscombe en el pasaje anteriormente citado del $\S 4$, pensar que debemos mirar "al interior" del agente o bien preguntarle por aquello que su acción expresa y que solo él, en último término, conoce. En último término, parece ocurrir que clasificamos como acción aquello ante lo cual podemos dar una explicación en términos del "propósito" que perseguimos. Pero esto no aclara todavía bien el asunto, al menos no mientras no sea claro qué es un propósito. Parece ser que, en el ejemplo de Anscombe, aceptamos que alguien que bombea agua puede estar "bombeando agua" o "envenenando a los habitantes de la casa", pero resulta más difícil de aceptar que él estime estar "instaurando el gobierno de los sabios". No podemos aquí elucidar esta cuestión en detalle; sin embargo, sí se puede hacer notar lo siguiente: no parece ser posible comprender la idea misma del propósito sin remitir a relaciones del tipo de aquellas que revisten el fin y los medios, de suerte que ella no queda entregada al mero reporte del agente. Esas relaciones, sin embargo, parecen ser relaciones de naturaleza causal (sea que el fin sea considerado en algún sentido como "causa" del medio o viceversa). Lo anterior sugiere que las relaciones entre fines y medios, que parecen ser constitutivas de la posibilidad de las acciones, implican la existencia de un orden de vínculos entre ítems que, en algún sentido, es independiente de mi pura interioridad. Dicho de otro modo: no cualquier acción puede ser considerada como siendo "medio" para 
alcanzar un fin que yo mismo declare como objetivo de esa acción. Así, nadie que me vea haciendo ejercicio en un gimnasio podría aceptar la descripción "estoy comiendo para subir de peso" como una que adecuadamente de cuenta del contenido de lo que hago. La razón es simple: la trotadora es un medio para correr y correr no es medio para subir de peso (así como correr tampoco es ingerir alimento). Lo anterior muestra, además, el modo en que esas relaciones causales están insertas en un orden cuerpos y de útiles que tienen carácter ergonómico, i.e. están diseñados para ser adecuados a los efectos esperados por medio de la realización de ciertas acciones, i.e. de ciertos movimientos corporales que bajo alguna descripción cuentan como intencionales. La trotadora tiene la forma de algo que se presta para correr (y para bajar peso corriendo en ella, pero no parece ser adecuada para comérsela). Lo mismo el ordenador que uso mientras escribo esto (su pantalla está dispuesta frente a mí y no debajo del teclado ni en la superficie trasera de la tapa, que no es visible desde mi posición actual). Este doble carácter de los útiles requeridos para la acción, sc. su capacidad de conectarse causalmente entre sí según reglas independientes de mí y la ergonomía, parecen suponer un orden en el cual la función del cuerpo propio es crucial como elemento básico en la constitución del sentido de estos entes.

Con todo hasta acá, se podría pensar, no se ha dicho aún nada que desmienta la rectitud de la presentación del problema de la espacialidad hecha por Heidegger. Es cierto, se podría decir, que es necesario el orden de los cuerpos para la acción, pero, tal como ya se advirtió, la experimentación de esos cuerpos como tales supone, se podría decir con Heidegger, por una parte, una ocupación desalejante y orientada del espacio, y, por otra, un mundo que ya se ha abierto ahí en la forma de zonas para esta orientación pragmática, constituyendo esta ocupación desalejante y orientada la estructura ontológica básica de la existencia, i.e. una estructura ontológica que constituye el abrir del Dasein el mundo independiente de lo que haga cada Dasein en particular. Es esta idea, con todo, la que está detrás de las relaciones de fundamentación entre los dos "espacios" que ya han sido mencionadas y que parecen en alguna medida dar cuenta del carácter a priori de la espacialidad del Dasein. Esta "fundaría", según Heidegger, el espacio geométrico. Pero la pregunta clave parece ser si es posible realmente fundar, como lo intenta hacer Heidegger, el orden de los cuerpos en 
esa ocupación desalejante y orientada en el espacio sin remitir antes al orden de los mismos y al modo en que ellos parecen vincularse según leyes objetivas (como la de los vínculos causales entre ellos). Y es aquí donde el asunto se vuelve difícil, especialmente porque no parece haber fundamento a partir del cual nos tengamos que ver constreñidos a asumir las relaciones de prioridad antedichas, tal como las presenta Heidegger. Es más, parece haber buenas razones más bien en contra de esta idea. En efecto, cuando intentamos comprender por qué la ocupación desalejante y orientada es anterior a la espacialidad en la que situamos nuestros cuerpos, Heidegger parece tratar la misma como si ella pudiese ser comprendida sin apelación al orden de los cuerpos, lo cual pareciera, por otra parte, dar cuenta del carácter fundante de esta forma de espacialidad. Con todo, cuando Heidegger trata de dar cuenta de estas relaciones de prioridad suele hacerlo a la luz de ejemplos que más bien parecen presentar percepciones de índole psíquica, que difícilmente parecen poder fundar lo que en la tradición angloamericana de comentadores de Heidegger se ha dado en llamar como "el carácter público del espacio" ${ }^{17}$. El ejemplo más claro de lo anterior parece ser dado por el modo en que Heidegger trata la cuestión de la "cercanía". Indica Heidegger aquí:

La orientación primaria e incluso excluyente en la lejanía como distancias medidas oculta la espacialidad originaria del ser-en. Lo supuestamente «más cercano» no es para nada lo que está a la distancia más corta «de nosotros«. Lo «más cercano» está en lo que está desalejado a mediano alcance, a la mediana distancia de tomarse o de verse. Dado que el Dasein es esencialmente espacial en el modo de la des-alejación, el trato se mantiene siempre en un «ambiente» desalejado por él siempre en un cierto ámbito. Por ello siempre oímos y vemos saltándonos lo «más cercano» desde el punto de vista de la distancia. Ver y oír son sentidos de la distancia no a causa de su mayor alcance, sino porque el Dasein está preponderantemente en ellos como desalejante. Por ejemplo, para el que usa anteojos ellos están desde el punto de vista de la distancia más cercanos, al punto de que los «tiene en la nariz», pero este útil usado está en el

La relevancia de estos ejemplos y el modo en que ellos presentan una enorme dificultad para las relaciones de prioridad sugeridas por Heidegger es mostrada con maestría por Cerbone (2013). 
mundo circundante más lejos que el cuadro en la pared de al frente. Este útil tiene tan poca cercanía, que usualmente ni siquiera se lo encuentra. El útil para ver, de la misma manera que aquel para oír, por ejemplo, el auricular del teléfono, tiene la ya indicada característica de lo a la mano de no llamar la atención (\$23 106-107).

Estos ejemplos, que han sido brillantemente analizados por Cerbone, así como el que sigue, en el que Heidegger realiza el mismo ejercicio de mostrar la lejanía circunmundana de aquello que desde el punto de vista "métrico" es lo más cercano, muestran que Heidegger parece ver en lo que podría denominarse como la "cercanía atencional" la forma primaria de cercanía espacial a partir de la cual deberían "derivarse" las eventuales restantes. Con todo, no resulta muy claro cómo puede ocurrir esto, incluso ni siquiera si ambos órdenes, tal como argumenta Cerbone, no terminan oponiéndose (Cerbone 141).

Aunque se podría sostener que este modo de comprender la cuestión confunde la "prioridad quoad nos" con la "prioridad en si" (si es que cabe una distinción de este tipo aquí), es claro que si, por un lado, la fijación del lugar que el agente de facto ocupa ("mi aquí") es dependiente de aquello con lo que ese agente se ocupa (el plexo de útiles del caso), y, por otra parte, ya el sentido mismo de actuar con el concurso de esos entes dispuestos para la acción implica algún tipo de relación entre cuerpos, entonces la "espacialidad del Dasein", en lo que refiere precisamente al "aquí" del agente, refiere constitutivamente a aquello cuya problemática los análisis de Heidegger eluden o al menos postergan, i.e, el propio cuerpo. Esta postergación parece quedar del todo clara en el hecho de que, en el recurso a los ejemplos, Heidegger no puede sino trasladar el énfasis al exponer el modo en que concibe las relaciones espaciales hacia ejemplos de "cercanía atencional", que parecen, por otro parte, dejar de lado el tipo de relación práctico-operativa con el ente que había sido ganada en los parágrafos previos y que constituyen el eje del tratamiento heideggeriano del útil, tratamiento que no supone, sino que de hecho más bien niega, como ha acreditado Cerbone, el que deba existir un requerimiento "atencional" para que éste tenga lugar (y es patente, además, a partir del mismo pasaje recién citado: "El útil para ver, de la misma manera que aquel para oír, por ejemplo el auricular del teléfono, tiene la ya indicada característica de lo a la mano de no llamar la atención"). 
Vista así, la reconstrucción del espacio realizada por Heidegger en los textos en comento (así como su concepción del útil) parecen dejar un lugar vacío, que necesariamente debería haber sido llenado si se toman en serio los mismos requerimientos metodológicos y sistemáticos de Heidegger. Por ello, se abre aquí un espacio para una crítica inmanente al planteo de SZ en torno a estas cuestiones que, más allá de implicar una crítica general a su obra, intenta dar cuenta de una tensión interna que ella presenta. No hay dudas, nos parece, de que esta tensión puede ser productiva, como probablemente lo fue para el mismo Heidegger. $Y$ aunque si bien hay indicios en obras posteriores de que Heidegger abandonó el planteo de la espacialidad realizado en SZ, lo cual hace suponer que presentó elementos insatisfactorios incluso para el mismo Heidegger, la genuina comprensión de la naturaleza de estos elementos supone, nos parece, un esfuerzo como el que hemos intentado realizar aquí. Ulteriores investigaciones deberían poder determinar si, siguiendo nuestra hipótesis, las eventuales reparaciones que Heidegger habría hecho a su concepción de la espacialidad, en obras que sucedieron a SZ, permiten realmente dejar de lado las objeciones aquí formuladas. Pero esto no puede sino ser tarea para otro trabajo.

\section{Bibliografía}

Aho, Kevin. Heidegger's Neglect of the Body. Albany: SUNY, 2009.

Anscombe, Gertrude Elizabeth Margaret. "Under a Description”, Noûs 13.2 (1979): 219-233.

Anscombe, Gertrude Elizabeth Margaret. Intention. Cambridge, MA: Harvard University Press, 2000.

Arisaka, Yoko. "Heidegger's Theory of Space: A Critique of Dreyfus", Inquiry 38.4 (1995): 455-467.

Cerbone, David. "Heidegger on Space and Spatiality". The Cambridge Companion to Being and Time. Mark A. Wrathall, editor. Cambridge: CUP, 2013. 129-144.

Davidson, Donald. Essays on actions and events, Oxford: OUP, 2001.

Dreyfus, Hubertus. Being-in-the-world. Cambridge, MA: MIT Press, 1991.

Franck, Didier. Heidegger et le Problème de l'espace. Les Éditions de Minuit: Paris, 1986. 
Heidegger, Martin. Prolegomena zur Geschichte des Zeitsbegriffs. Frankfurt am Main: Vittorio Klostermann, 1979 (=GA 20).

Heidegger, Martin. Sein und Zeit. Tübingen: Max Niemeyer, 2001 (=SZ).

Heidegger, Martin. Zur Sache des Denkens. Frankfurt am Main: Vittorio Klostermann, 2007 (=GA 14).

Hyman, John. Action, knowledge and will. Oxford: OUP, 2015.

Kant, Immanuel. Kant's gesammelte Schriften. Herausgegeben von der Preussischen bzw. von der, Berlin: Reimer, 1902-2009

Kant, Immanuel. Kritik der reinen Vernunft. Hamburg: Felix Meiner, $1956(=\mathrm{KrV})$.

Kisiel, Theodore. "On the Way to Being and Time; Introduction to the Translation of Heidegger's. Prolegomena zur Geschichte des Zeitsbegriffs", Research in Phenomenology 15.1 (1985): 193219.

Placencia, Luis. La ontología del espacio en Kant. Pamplona: Cuadernos de Anuario Filosófico, 2007.

Placencia, Luis. Handlung und praktisches Urteil bei Kant. FreiburgMünchen: Karl Alber, 2019.

Vallega, Arturo. Heidegger and the Issue of Space. Pennsylvania: Pennsylvania State University, 2003.

Villela-Petit, Maria. "Heidegger's Conception of Space". Martin Heidegger. Critical Assessments. Christopher Macann, editor. London-New York: Routledge, 1992.

Wright, G. H. von. Explanation and Understanding. Ithaca: Cornell University Press, 1971. 